\title{
Gestión de riesgos en proyectos de tecnología de información en el Perú
}

\begin{abstract}
RESUMEN
El presente artículo muestra algunos principios, usos y prácticas de cómo la gestión de riesgos de proyectos de tecnología se ha llevado a cabo en los últimos cuatro años en el Perú, en sectores representativos como manufactura, banca, información y comunicaciones, instituciones académicas, construcción, gobierno, consultoría, servicios, y otros.
\end{abstract}

Palabras clave: Gestión de riesgos, proyectos tecnología de información

INFORMATION TECHNOLOGY PROJECT RISK MANAgement in Peru

\section{ABSTRACT \\ This article shows how some principles, uses, and practices of risk management are applied in infor- mation technology projects in Peru; in the last four years, in representative sectors like manufacturing banking, information and communications, acade- mics institutions, construction, government, con- sulting, services, and others.}

Key words: Risk management, projects, and information technology.

\section{INTRODUCCIÓN}

En mayo de 2006, se publicó el artículo Análisis de Riesgos en la Administración de Proyectos de Tecnología (Del Carpio, 2006)², señalando la importancia del tema y recomendando que se continuara investigando sobre la gestión de riesgos. Se consideró de esa manera por que el tema ha sido tratado por distintos académicos, como por ejemplo los Doctores Chapman y Ward (2003), que establecen3: "La gestión de riesgos de los proyectos está generalmente asociada con el desarrollo y evaluación de planes de contingencia que dan soporte a los planes de actividades, pero la gestión de riesgos de proyectos efectiva será instrumental en el desarrollo de planes de base y planes de contingencia para cada una de las seis interrogantes:

- ¿Quiénes son los involucrados? (Involucrados)

- ¿Qué es lo que lo se quiere lograr? (Motivos)

- ¿En qué están interesados los involucrados? (Diseño)

- ¿Cómo será hecho? (Actividades)

- ¿Qué recursos son requeridos? (Recursos)

- ¿Cuándo tiene que ser hecho? (Programación)

La administración de riesgos de los proyectos ha sido abordada por instituciones tan prestigiosas como Project Management Institute (2004), que en su libro A Guide to the Project Management Body of Knowledge ${ }^{4}$ (PMBOK) establece que "la gestión de riesgos de los proyectos incluye los procesos concernientes con la conducción de la planificación, identificación, análisis, respuesta, monitoreo y control de un proyecto."

Según el PMBOK, la gestión de los riesgos del proyecto ${ }^{5}$ considera seis actividades:

- Planificación

- Identificación

- Análisis cualitativo

- Análisis cuantitativo

- Planificación de la respuesta

- Monitoreo y control

Pero no solamente académicos y organizaciones profesionales han tratado sobre la gestión de riesgos de proyectos de TI; sino también empresas privadas como Microsoft Corporation, consideran que la Gestión

M. Sc. Docente de la Universidad Nacional Mayor de San Marcos. jdecarpiog@unmsm edu.pe Del Carpio Gallegos, Javier F. (2006). Análisis del Riesgo en la Administración de Proyectos de Tecnología de Información. Industrial Data, Vol. 9, ‥ ${ }^{\circ} 1$, pp. 104-107.

3 Chapman, Chris \& Ward, Stephen. (2003). Project Risk Management. Second Edition. John Wiley and Sons. pp. 15.

4 Project Management Institute, A Guide to the Project Management Body of Knowledge (PMBOK Guide) Third edition. 2004, pp. 45.

5 Ibid., pp. 242. 
de Riesgos es una disciplina fundamental, elaborando lo que se conoce como Microsoft Solutions Framework (MSF). MSF reconoce que el cambio y la incertidumbre son aspectos inherentes del ciclo de vida de la tecnología de información. La disciplina de Gestión de Riesgos de MSF recomienda un enfoque proactivo para tratar con esta incertidumbre, evaluando los riesgos continuamente, y usándolos para influenciar la toma de decisiones a lo largo del ciclo de vida. La disciplina describe principios conceptos, y guías en un proceso de cinco pasos para lograr una gestión de riesgos exitosa y permanente: identificar riesgos, analizar riesgos, planear contingencias y estrategia de mitigación, controlar el estatus de los riesgos, y aprender de los resultados ${ }^{6}$.

También, son reconocidos estándares de otros continentes como el estándar ASS/NZS 4360: 2004 Gestión de Riesgos (Australia/New Zealand Standard). Este estándar fue preparado por la Joint Standards Australia/Standards New Zealand Comité OB007, proporcionando una guía genérica para gestionar riesgos; pudiendo ser aplicado a una amplia variedad de actividades, decisiones u operaciones de cualquier empresa, grupo, o individuo público o privado; en tanto que el estándar tiene una amplia aplicabilidad, los procesos de gestión de riesgos son comúnmente aplicados por organizaciones ${ }^{7}$.

Por otro lado, las organizaciones europeas han establecido desde 1998, el Instituto de Gobierno de Tecnología de Información (IT Governance Institute www.itgi.org) para lograr el avance del pensamiento internacional y estándares en la dirección y control de la tecnología de la información de una organización. El Instituto de Gobierno de Tecnología de Información ha diseñado y creado la publicación llamada COBIT 4.O (Control Objectives Management Guidelines Maturity Models), principalmente como un recurso educativo para los Gerentes de Tecnología de Información, alta dirección, y profesionales de la administración de Tecnología de Información. El esquema COBIT tiene 34 procesos genéricos los cuales permiten administrar los recursos de Tecnología de Información para entregar información a la organización de acuerdo a los requerimientos del negocio. Uno de los 34 procesos corresponde al proceso de evaluar y administrar los riesgos ${ }^{8}$.

\section{Aplicación de la encuesta}

Para poder saber como la gestión de riesgos es aplicada en los proyectos de tecnología de información en el Perú, en sectores tan representativos como manufactura, banca, información y comunicaciones, instituciones académicas, construcción, gobierno, consultoría, servicios, y otros; se realizó una encuesta consistente en 35 preguntas, la mencionada encuesta se puede consultar en el Campus Virtual de la Facultad de Ingeniería Industrial de la UNMSM (Visitar http://campusvirtualfii.unmsm.edu.pe/encuesta/). Para obtener la información, se remitió un mensaje electrónico a docentes de universidades, profesionales vinculados a colegios profesionales, y egresados de nuestra Casa de Estudios, obteniéndose en el periodo de octubre y noviembre de 2008, las respuestas de 30 encuestados. El bajo número de personas que han respondido las encuestas se debe a la premisa de exigir que los encuestados cumplan con la condición de ser gerentes o miembros de proyectos de tecnología de información

\section{Análisis de la información proporcionada por las encuestas}

Se ha realizado el análisis de 12 de las 35 preguntas formuladas, se ha elegido las preguntas más representativas, quedando pendiente para más adelante el análisis de las preguntas restantes.

Pregunta: ¿En cuántos proyectos de tecnología de información (TI) ha participado dese el 1 de enero de 2005 hasta al $\mathbf{3 0}$ de setiembre de 2008 ?

En el Cuadro N. ${ }^{\circ}$ 1: Participación en Proyectos de $\mathrm{TI}$, el cual muestra la cantidad de proyectos de tecnología de información en los cuales han participado los encuestados en el periodo comprendido entre el 1 de enero de 2005 al 30 de setiembre de 2008 .

Cuadro N. 1 Participación en Proyectos de TI

\begin{tabular}{|c|c|c|}
\hline $\begin{array}{c}\text { Rango } \\
\text { (Núm. de Proyectos) }\end{array}$ & Frecuencia & $\begin{array}{c}\text { Porcentaje } \\
(\%)\end{array}$ \\
\hline $1-5$ & 15 & 50,0 \\
\hline $6-10$ & 10 & 33,3 \\
\hline $11-15$ & 2 & 6,7 \\
\hline Más de 15 & 3 & 10,0 \\
\hline Total & 30 & 100,0 \\
\hline
\end{tabular}

FUENTE: Elaboración propia.

Como se puede apreciar en el cuadro anterior, el $50 \%$ de los encuestados ha participado, por lo menos, en un proyecto, y más de $33 \%$ ha participado en más de seis proyectos; lo cual demuestra que los encuestados cuentan con experiencia en la planificación, ejecución y control de proyectos de TI.

\footnotetext{
6 Microsoft Solutions Framework. (2002) MSF Risk Management Discipline v. 1.1 p. 4.

7 ASS/NZS 4360:2004 Risk Management (Australia/New Zealand Standard). p. 1

8 IT Governance Institute. (2005). COBIT 4.0. p. 5
} 
Pregunta: ¿Cuál es su posición actual dentro de vuestra organización?

En el Cuadro N. ${ }^{\circ}$ 2: Posición actual dentro de la organización, el cual muestra la posición o cargo que desempeñan los encuestados

\section{Cuadro N. ${ }^{\circ}: 2$ Posición actual dentro de la organización}

\begin{tabular}{|c|c|c|}
\hline Posición & Frecuencia & $\begin{array}{c}\text { Porcentaje } \\
\text { (\%) }\end{array}$ \\
\hline Ejecutivo Senior & 5 & 16,7 \\
\hline Gerente de Proyecto & 17 & 56,7 \\
\hline $\begin{array}{c}\text { Miembro del equipo } \\
\text { del proyecto }\end{array}$ & 8 & 26,7 \\
\hline Total & 30 & 100,0 \\
\hline
\end{tabular}

FUENTE: Elaboración propia

Como se puede apreciar en el cuadro anterior, más de $56 \%$ de los encuestados se ha desempeñado como Gerente de proyecto de tecnología de información; lo cual demuestra que la mayoría de los encuestados han realizado la gestión de riesgos desde un punto de vista integral, es decir, relacionado los riesgos con los demás procesos de la gestión de proyectos como son: la estimación de costos y plazos, la gestión de recursos humanos, de aprovisamiento, comunicación, control de calidad, entre otros.

Pregunta: Tipo de sector o industria al cual pertenece vuestra organización

En el Cuadro N. ${ }^{\circ}$ 3: Tipo de sector al cual pertenecen las organizaciones de los encuestados, el cual muestra el tipo de actividad desarrollada por la organización.

Cuadro N. ${ }^{\circ} 3$ Tipo de sector al cual pertenecen las organizaciones de los encuestados

\begin{tabular}{|c|c|c|}
\hline Alternativas & Frecuencia & $\begin{array}{c}\text { Porcentaje } \\
\text { (\%) }\end{array}$ \\
\hline $\begin{array}{c}\text { Información y } \\
\text { comunicaciones }\end{array}$ & 6 & 20,0 \\
\hline Banca & 5 & 16,7 \\
\hline Institución académica & 4 & 13,3 \\
\hline Manufactura & 2 & 6,7 \\
\hline Construcción & 2 & 6,7 \\
\hline Gobierno & 2 & 6,7 \\
\hline Consultoría & 1 & 3,3 \\
\hline Servicios & 1 & 3,3 \\
\hline Otros & 7 & 23,3 \\
\hline Total & 30 & 100,0 \\
\hline
\end{tabular}

FUENTE: Elaboración propia.
Como se puede apreciar en el cuadro anterior, los sectores más representativos son los de informaciones y comunicaciones, y banca. Pero se puede apreciar que los proyectos de tecnología de información se desarrollan en una diversidad de actividades empresariales e institucionales.

Pregunta: ¿Cuántos años tiene de experiencia en gestión de proyectos de tecnología de la información?

En el Cuadro N.$^{\circ} 4$ : Años de experiencia de los encuestados, el cual muestra el número de años de experiencia en la gestión de proyectos de tecnología de información de los encuestados.

\section{Cuadro N. ${ }^{\circ}$ 4: Años de experiencia de} los encuestados

\begin{tabular}{|c|c|c|}
\hline $\begin{array}{c}\text { Experiencia } \\
\text { (Num. de años) }\end{array}$ & Frecuencia & $\begin{array}{c}\text { Porcentaje } \\
\text { (\%) }\end{array}$ \\
\hline $1-5$ & 11 & 36,7 \\
\hline $6-10$ & 13 & 43,3 \\
\hline $11-15$ & 4 & 13,3 \\
\hline Mas de 15 & 2 & 6,7 \\
\hline Total & 30 & 100,0 \\
\hline
\end{tabular}

FUENTE: Elaboración propia

Como se puede apreciar en el cuadro anterior, más de $63 \%$ de los encuestados tiene por lo menos seis años de experiencia participando en proyecto de tecnología de información. Lo cual demuestra que los encuestados tiene la experiencia deseable participar en proyectos de TI.

Pregunta: Ingresos anuales de vuestra organización durante el año 2007 (Cantidad en dólares americanos)

En el Cuadro N. ${ }^{\circ}$ 5: Ingresos anuales en 2007 (Cifras en dólares americanos), el cual muestra el rango de los ingresos anuales de las organizaciones a las cuales pertenecen los encuestados. Como se puede apreciar en el cuadro anterior, las organizaciones encuestas van desde las pequeñas empresas hasta las grandes empresas. Asimismo, más de $53 \%$ de las organizaciones de los encuestados tuvieron en el año 2007, ingresos de por lo menos 15 millones de dólares americanos.

Pregunta: ¿Su organización ha implementado una política de gestión de riesgos de proyectos de tecnología de la información (TI)?

En el Cuadro N. ${ }^{\circ}$ 6: Implementación de política de gestión de riesgos en Proyectos de TI (Tecnología 
de Información), el cual muestra el número de organizaciones que aplican dicha política.

\section{Cuadro N. ${ }^{\circ}$ 5: Ingresos anuales en 2007} (Cifras en US\$)

\begin{tabular}{|c|c|c|}
\hline $\begin{array}{c}\text { Rango } \\
\text { (Ingresos anuales) }\end{array}$ & Frecuencia & $\begin{array}{c}\text { Porcentaje } \\
(\%)\end{array}$ \\
\hline Hasta US\$ 350.000 & 3 & 10,0 \\
\hline $\begin{array}{c}\text { Desde US } \$ 350.001 \\
\text { hasta US\$ 3.000.000 }\end{array}$ & 7 & 23,3 \\
\hline $\begin{array}{c}\text { Desde US\$ 3.000.001 } \\
\text { hasta US\$ 15.000.000 }\end{array}$ & 4 & 13,3 \\
\hline $\begin{array}{c}\text { Desde US\$ 15.000.001 } \\
\text { hasta US\$ 30.000.000 }\end{array}$ & 4 & 13,3 \\
\hline $\begin{array}{c}\text { Desde US\$ 30.000.001 } \\
\text { hasta US\$150.000.000 }\end{array}$ & 2 & 6,7 \\
\hline $\begin{array}{c}\text { Más de } \\
\text { US 150.000.000 }\end{array}$ & 10 & 33,3 \\
\hline Total & 30 & 100,0 \\
\hline
\end{tabular}

FUENTE: Elaboración propia.

Cuadro N. 6: Implementación de política de gestión de riesgos en Proyectos de $\mathrm{TI}$

\begin{tabular}{|c|c|c|}
\hline Alternativas & Frecuencia & Porcentaje (\%) \\
\hline Sí & 13 & 43,3 \\
\hline No & 15 & 50,0 \\
\hline No sabe & 2 & 6,7 \\
\hline Total & 30 & 100,0 \\
\hline
\end{tabular}

FUENTE: Elaboración propia.

Como se puede apreciar en el cuadro anterior, más de 43 por ciento de las organizaciones a las cuales pertenecen los encuestados tienen implementado una política de gestión de riesgos en el desarrollo de proyectos de tecnología de información.

Pregunta: ¿Si su respuesta fue afirmativa en la pregunta anterior, en que año vuestra organización implemento una política de gestión de riesgos de proyectos de tecnología de la información (TI)?

En el Cuadro N. ${ }^{\circ}$ 7: Antigüedad de la implementación de política de gestión de riesgos en Proyectos de TI (Tecnología de Información), el cual muestra el numero de años que las organizaciones encuestadas tienen implementada una política de gestión de riesgos.

Como se puede apreciar en el cuadro anterior, más de $69 \%$ de las organizaciones a que han implementado una política de gestión de riesgos en el desarrollo de proyectos de tecnología de información, lo implementaron antes de 2005. Lo cual demuestra que la gestión de riesgos de proyectos de tecnología de información es un tema que demando atención en, por lo menos, los últimos 4 años.

Cuadro N..$^{7}$ : Antigüedad de la implementación de política de gestión de riesgos en Proyectos de TI

\begin{tabular}{|c|c|c|}
\hline Alternativas & Frecuencia & Porcentaje (\%) \\
\hline Antes del 2005 & 9 & 69,2 \\
\hline 2005 & 0 & 0,0 \\
\hline 2006 & 1 & 7,7 \\
\hline 2007 & 1 & 7,7 \\
\hline 2008 & 2 & 15,4 \\
\hline Total & 13 & 100,0 \\
\hline
\end{tabular}

FUENTE: Elaboración propia.

Pregunta: ¿Los ejecutivos senior de vuestra organización proporcionan los recursos financieros, humanos, y los plazos necesarios para conducir el proceso de gestión de riesgos de los proyectos de tecnología de la información (TI)?

En el Cuadro N. ${ }^{\circ}$ 8: Disposición de recursos para conducir el proceso de gestión de riesgos en proyectos de TI., el cual muestra el número de las organizaciones encuestadas que proporciona los recursos necesarios.

Cuadro N. 8: Disposición de recursos para conducir el proceso de gestión de riesgos en Proyectos de TI

\begin{tabular}{|c|c|c|}
\hline Alternativas & Frecuencia & Porcentaje (\%) \\
\hline Raramente (1-19\%) & 9 & 30,0 \\
\hline $\begin{array}{c}\text { Ocasionalmente } \\
(20-39 \%)\end{array}$ & 5 & 16,7 \\
\hline $\begin{array}{c}\text { Frecuentemente } \\
(40-59 \%)\end{array}$ & 8 & 26,7 \\
\hline $\begin{array}{c}\text { Generalmente } \\
(60-79 \%)\end{array}$ & 1 & 3,3 \\
\hline $\begin{array}{c}\text { Casi siempre } \\
(80-100 \%)\end{array}$ & 3 & 10,0 \\
\hline No sabe & 30 & 13,3 \\
\hline Total & \multicolumn{2}{|c|}{} \\
\hline
\end{tabular}

FUENTE: Elaboración propia.

Como se puede apreciar en el cuadro anterior, $40 \%$ de las organizaciones a las que pertenecen los encuestados cuenta por lo menos frecuentemente con los recursos necesarios para realizar la gestión de riesgos. Esta información llama la atención, pues si no se proporcionan los recursos necesarios, la probabilidad de no cumplir con los objetivos de costos, plazo, y calidad se incrementa. 
Pregunta: ¿Los gerentes de proyectos usan alguna herramienta cuantitativamente de gestión de riesgos? (Simulación de Monte Carlo, Árboles de decisiones, Análisis de escenarios)

En el Cuadro N. ${ }^{\circ}$ 9: Utilización de herramientas cuantitativas en el proceso de gestión de riesgos en Proyectos de $\mathrm{Tl}$, el cual muestra el número de las organizaciones encuestadas que hacen uso de herramientas cuantitativas de gestión de riesgos.

Cuadro $\mathrm{N}^{\circ}{ }^{\circ}$ 9: Utilización de herramientas cuantitativas en el proceso de gestión de riesgos en Proyectos de TI

\begin{tabular}{|c|c|c|}
\hline Alternativas & Frecuencia & Porcentaje (\%) \\
\hline $\begin{array}{c}\text { Raramente } \\
(1-19 \%)\end{array}$ & 17 & 56,7 \\
\hline $\begin{array}{c}\text { Ocasionalmente } \\
(20-39 \%)\end{array}$ & 1 & 3,3 \\
\hline $\begin{array}{c}\text { Frecuentemente } \\
(40-59 \%)\end{array}$ & 3 & 10,0 \\
\hline $\begin{array}{c}\text { Generalmente } \\
(60-79 \%)\end{array}$ & 2 & 6,7 \\
\hline $\begin{array}{c}\text { Casi siempre } \\
(80-100 \%)\end{array}$ & 1 & 3,3 \\
\hline No sabe & 6 & 20,0 \\
\hline Total & 30 & 100,0 \\
\hline
\end{tabular}

FUENTE: Elaboración propia.

Como se puede apreciar en el cuadro anterior, más de $56 \%$ de los encuestados raramente utiliza herramientas cuantitativas de gestión de riesgos. Y, apenas 3,3\% de los encuestados casi siempre las utiliza. Esto demuestra falta de conocimiento sobre la aplicación de las herramientas cuantitativas de gestión de riesgos.

Pregunta: ¿Los gerentes de proyectos usan alguna herramienta cualitativamente de gestión de riesgos (Matriz de probabilidad e impacto)?

En el Cuadro N. ${ }^{\circ}$ 10: Utilización de herramientas cualitativas en el proceso de gestión de riesgos en Proyectos de $\mathrm{TI}$, el cual muestra el número de las organizaciones encuestadas que hacen uso de herramientas cualitativas de gestión de riesgos.

Como se puede apreciar en el cuadro anterior, $40 \%$ de los encuestados por lo menos generalmente utiliza herramientas cualitativas de gestión de riesgos. Esto se condice con el hecho que las herramientas cualitativas son más conocidas y de mayor aplicación.

Pregunta: ¿Durante cual de las siguientes fases del desarrollo del proyecto de tecnología de la información (TI) se preparan los planes de contingencia para responder a riesgos específicos?
Cuadro $\mathrm{N}^{\circ}{ }^{\circ}$ 10: Utilización de herramientas cualitativas en el proceso de gestión de riesgos en Proyectos de TI

\begin{tabular}{|c|c|c|}
\hline Alternativas & Frecuencia & Porcentaje (\%) \\
\hline $\begin{array}{c}\text { Raramente } \\
(1-19 \%)\end{array}$ & 7 & 23,3 \\
\hline $\begin{array}{c}\text { Ocasionalmente } \\
(20-29 \%)\end{array}$ & 7 & 23,3 \\
\hline $\begin{array}{c}\text { Frecuentemente } \\
(30-59 \%)\end{array}$ & 2 & 6,7 \\
\hline $\begin{array}{c}\text { Generalmente } \\
(60-79 \%)\end{array}$ & 9 & 30,0 \\
\hline $\begin{array}{c}\text { Casi siempre } \\
(80-100 \%)\end{array}$ & 3 & 10,0 \\
\hline No sabe & 2 & 6,7 \\
\hline Total & 30 & 100,0 \\
\hline
\end{tabular}

FUENTE: Elaboración propia.

En el Cuadro N. ${ }^{\circ}$ 11: Identificación de las fases en las cuales se realiza el plan de contingencia, el cual muestra la fase del proyecto en la cual se realiza la preparación de los planes de contingencia.

Cuadro N. 11: Identificación de las fases en las cuales se realiza el plan de contingencia

\begin{tabular}{|c|c|c|}
\hline Fases & Frecuencia & Porcentaje (\%) \\
\hline Iniciación & 6 & 20,0 \\
\hline Planeación & 14 & 46,7 \\
\hline $\begin{array}{c}\text { Ejecución y } \\
\text { control }\end{array}$ & 8 & 26,7 \\
\hline Cierre & 1 & 3,3 \\
\hline No sabe & 1 & 3,3 \\
\hline Total & 44 & 100,0 \\
\hline
\end{tabular}

FUENTE: Elaboración propia.

Como se puede apreciar en el cuadro anterior, más del $46 \%$ de los encuestados preparara los planes de contingencia en la fase de planeación, y un $20 \%$ lo realiza desde la fase de inicio o concepción del proyecto. Lo cual se constituye en una buena practica, pues en la medida que los planes de contingencia se realicen con mayor anticipación, se tendrá mayor probabilidad de éxito para enfrentar a los riesgos.

Pregunta: ¿Usted considera que las políticas de gestión de riesgo de su organización hacen una diferencia medible del desempeño de los proyectos de tecnología de información?

En el Cuadro N. ${ }^{\circ}$ 12: Las políticas de gestión de riesgo de su organización hacen una diferencia medible del desempeño de los proyectos de tecnología de información, el cual muestra la opinión de los encuestados sobre la importancia de las políticas de riesgos. 
Cuadro N. 12 Las políticas de gestión de riesgo de su organización hacen una diferencia medible del desempeño de los proyectos de tecnología de información

\begin{tabular}{|c|c|c|}
\hline Alternativas & Frecuencia & Porcentaje (\%) \\
\hline $\begin{array}{c}\text { Raramente } \\
(1-19 \%)\end{array}$ & 5 & 16,7 \\
\hline $\begin{array}{c}\text { Ocasionalmente } \\
(20-29 \%)\end{array}$ & 6 & 20,0 \\
\hline $\begin{array}{c}\text { Frecuentemente } \\
(30-59 \%)\end{array}$ & 7 & 23,37 \\
\hline $\begin{array}{c}\text { Generalmente } \\
(60-79 \%)\end{array}$ & 4 & 13,3 \\
\hline $\begin{array}{c}\text { Casi siempre } \\
(80-100 \%)\end{array}$ & 3 & 10,0 \\
\hline No sabe & 5 & 16,7 \\
\hline Total & 30 & 100,0 \\
\hline
\end{tabular}

FUENTE: Elaboración propia.

Como se puede apreciar en el cuadro anterior, más del $63 \%$ de los encuestados considera que las políticas de gestión de riesgo de su organización hacen una diferencia medible del desempeño de los proyectos de tecnología de información. Es decir, una adecuada gestión de riesgos se traduce en proyectos dentro del plazo y presupuesto, cumpliendo con los requerimientos de los involucrados.

\section{CONCLUSIONES}

- La gestión de riesgos de proyectos de tecnología de información constituye un tema fundamental, el cual está siendo tratado en el medio académico, profesional e institucional en todo el mundo.

- La información obtenida de las encuestas, a pesar de no haber reunido información de un número significativo de encuestados. La información proporcionada es muy relevante tomando en cuenta que los encuestados son profesionales de gestión de proyectos con mucha experiencia tanto en tiempo como en el número de proyectos en los cuales han participado.

- La gestión de proyectos de tecnología de información se viene desarrollando en organizaciones que pertenecen a una diversidad de sectores económicos, y de diferente nivel de ingresos, pero particularmente en los sectores información, comunicaciones, y banca, sectores en los cuales existe un alto nivel de competencia.

- De las organizaciones encuestadas más del $43 \%$ tiene implementada una política de gestión de riesgos, de las cuales casi el $70 \%$ de las mismas implementó dichas políticas en los últimos cuatro años. Sin embargo, estas organizaciones no asignan los recursos financieros, humanos, y los plazos necesarios para la implementación adecuada de las mencionadas políticas de gestión de riesgo, lo cual tiene como consecuencia el incremento en los costos y el exceso en el cumplimento de los plazos de los proyectos de tecnología de información.

- Se puede apreciar que los responsables de los proyectos de tecnología de información no tienen el conocimiento adecuado de las herramientas cuantitativas (Simulación de Monte Carlos, Árboles de Decisión, entre otros) de gestión de riesgos, y se apoyan, generalmente, en las herramientas cualitativas (Matriz de probabilidad e impacto que se basan en la opinión o juicios de expertos)

- Más del $46 \%$ de las organizaciones encuestadas concentra la elaboración de los planes de contingencia para poder responder a los riesgos en la fase de planificación de los proyectos, lo cual se constituyen en una buena práctica, pues en la medida que se planteen medidas para poder enfrentar, o atenuar el impacto de los riesgos, se tendrá una mayor probabilidad de éxito en la gestión de los proyectos.,

- Más del $63 \%$ de los encuestados considera que las políticas de gestión de riesgo de su organización hacen una diferencia medible del desempeño de los proyectos de tecnología de información. Es decir, una adecuada gestión de riesgos se traduce en proyectos dentro del plazo y presupuesto, cumpliendo con los requerimientos de calidad y el alcance del proyecto.

\section{RECOMENDACIONES}

- Continuar investigando en el tema de gestión de riesgos orientado a los proyectos de tecnología de información. Debido a que las organizaciones públicas y privadas tiene que enfrentar un ambiente más cambiante y competitivo, y la tecnología d información tiende a convertirse en una ventaja competitiva significativa.

- Realizar estudios comparativos entre sectores de actividad empresarial, y tamaño de la organización.

- Constituir núcleos de investigación en torno al tema de la gestión de riesgos en los proyectos de tecnología de información.

- Difundir el uso y manejo de las herramientas cuantitativas entre los gerentes de proyectos de tecnología de información, que les permitirá lograr un mejor desempeño. 


\section{REFERENCIAS BIBLIOGRÁFICAS}

1. ASS/NZS 4360: 2004 Risk Management (Australia/New Zealand Standard).

2. Chapman, Chris \& Ward, Stephen (2003). Project Risk Management. Second Edition. John Wiley and Sons.

3. Del Carpio Gallegos, Javier F. (2006). Análisis del Riesgo en la Administración de Proyectos de
Tecnología de Información. Industrial Data, Vol. 9, N. ${ }^{\circ}$, pp. 104-107.

4. Microsoft Solutions Framework (2002). MSF Risk Management Discipline v. 1.1.

5. Project Management Institute (2004). A Guide to the Project Management Body of Knowledge (PMBOK Guide) Third edition.

6. IT Governance Institute (2005). COBIT 4.0. 